\title{
Correlation of blood culture and band cell ratio in neonatal septicaemia.
}

\author{
Nautiyal $S^{1}$., *Kataria V. K ${ }^{1}$, Pahuja V. $K^{1}$., Jauhari $S^{1}$., Roy R. C., ${ }^{1}$ \\ Aggarwal B. ${ }^{2}$ \\ ${ }^{I}$ Department of Microbiology, SGRRIM\&HS and SMIH, Dehradun, Uttarakhand, India. \\ ${ }^{2}$ Department of Paediatrics, SGRRIM\&HS and SMIH, Dehradun, Uttarakhand, India.
}

\begin{abstract}
Background: Neonatal sepsis is a clinical syndrome characterized by signs and symptoms of infection with or without accompanying bacteraemia in the first month of life. Incidence differs among hospitals depending on variety of factors. Blood culture is considered gold standard for the diagnosis, but does not give a rapid result. Hence, there is a need to look for a surrogate marker for diagnosing neonatal septicaemia. Material \& Methods: 335 neonates were studied for clinically suspected septicaemia over a period of one year. Blood was cultured and organism identified biochemically. Parameters of subjects like EOS, LOS and Band cell counts were recorded. Results analysed statistically. Results: Male preponderance was observed. Majority of the cases had a normal vaginal delivery. $47.46 \%$ cases had early onset septicaemia. Meconium stained liquor was the predominant risk factor.Culture positivity was found to be $32.24 \%$ and $87.96 \%$ of them also had band cells percentage ranging from 0 to $>25$. Conclusion: Band cell count can be used as a surrogate marker for neonatal septicaemia. An upsurge of Candida species as a causative agent in Neonatal septicaemia has been observed.
\end{abstract}

\section{Introduction:}

Neonatal sepsis is a clinical syndrome characterized by signs and symptoms of infection with or without accompanying bacteraemia in the first month of life. It encompasses various systemic infections of the newborn such as septicemia, meningitis, pneumonia, arthritis, osteomyelitis and urinary tract infections. Superficial infections like conjunctivitis and oral thrush are not usually included under neonatal sepsis. (1)

According to the data from National Neonatal Perinatal Database (NNPD, 2002-03) the incidence of neonatal sepsis is 30 per 1000 live births. The database comprising 18 tertiary care neonatal units across India found sepsis to be one of the commonest causes of neonatal mortality contributing to $19 \%$ of all neonatal deaths.(2)

Infection is the primary cause of mortality in $18.6 \%$ of intramural neonates and $38 \%$ of extramural babies. Klebsiella pneumoniae being the most frequent bacterial isolate $32.5 \%$ and $27.5 \%$ in both intramural and extramural admissions respectively. Followed by Staphylococcus aureus in intramural (13.6\%) and extramural (14.9\%) admissions.(2) A rising trend of the role of Candida species in the pathogenicity of neonatal sepsis has also been observed by several workers.(3-7)

Incidence differs among hospitals depending on various factors such as obstetric and nursery practices, perinatal care, health and nutrition of mother and incidence of prematurity.

Though a positive blood culture is the gold standard for the diagnosis of neonatal septicaemia, it does not provide a rapid diagnosis. $(3,8)$ Hence, there is a need to look for a reliable surrogate marker for neonatal septicaemia to start an empirical treatment till culture report is awaited. (8)

The band cell ratio (ratio of mature to immature neutrophils) can be included in rapid diagnosis of neonatal septicaemia. The ratio is considered increased when it is more than 0.2 (Normal Band cell ratio is 0.030.05). Band neutrophil counts are helpful for the detection of neonatal sepsis. Modern automated hematology instruments provide an accurate and precise total neutrophil count but do not report band numbers which need to be calculated manually. $(1,9,10)$

Our goal was to detect elevated band neutrophils whenever present and correlate it with blood cultures from NICU in the suspected cases of neonatal sepsis admitted in our hospital (Shri Mahant Indiresh Hospital, Dehradun).

\section{Study design:}

In this prospective study blood samples from 335 neonates admitted for clinically suspected neonatal sepsis in the NICU in SMIH, Dehradun were collected, from January 2012 to December 2012. 


\section{Selection criteria for Subjects \\ Inclusion criteria:}

1. Neonates of both sexes were included in this study.

2. Neonates presenting with signs and symptoms such as refusal to feed, lethargy, fever, hypothermia, vomiting, diarrhoea, abdominal distension, jaundice, respiratory distress, seizures, or any external evidence of sepsis like umbilical cord infection, skin infection etc. were taken up for study.

3. A sample showing the growth of organisms of low pathogenicity, on repeat culture was included in this study.

\section{Exclusion criteria:}

1. Neonates with absence of signs of sepsis were excluded from this study.

2. Low pathogenic organisms like CoNS, Candida spp. unless grown on repeat culture were excluded.

\section{Material and methods:}

BacT/ALERT automated culture system was used for blood culture.

Positive culture bottles were promptly removed and a smear was made for Gram staining. Then a subculture was done on Columbia blood agar and MacConkey Agar and incubated aerobically at $37^{\circ} \mathrm{C}$ for $16-18$ hrs. After 18 hours plates were observed for growth and colonies were processed according to standard Microbiological procedures. $(11,12)$

Antibiotic sensitivity was done by Kirby Bauer's disc diffusion method as per CLSI guidelines. (13)

Total \& differential neutrophil counts were performed in Beckman Coulter automated system and Band cell ratio was calculated manually.

\section{Statistical analysis:}

Results were analysed using Chi square method for statistical significance.

\section{Results:}

A total of 335 neonates were studied for clinically suspected septicaemia. Male preponderance was observed with 213/335 (63.55\%) males and 122/335 (36.41\%) females. 192/335 (57.31\%) neonates had a normal vaginal delivery, 134/335 (40\%) of the neonates were delivered by LSCS. Other methods including instrumentation and vacuum assisted delivery were $9 / 335$ (2.68\%). So, majority of our study population had a normal vaginal delivery.

Early onset septicaemia was observed clinically in 159/335 (47.46\%) neonates, of which 36/159 (22.64\%) were culture positive. While 176/335 (52.53\%) had a late onset of septicaemia and 72/176 (40.90\%) were culture positive. Statistically this finding was highly significant $(\mathrm{P}=0.0004)$. (Table 1$)$

When various risk factors were analysed we found that meconium stained liquor (MSL) dominated followed by premature rupture of membranes (PROM), mechanical ventilator support (MVS) and twin deliveries. P value was highly significant when compared with clinically suspected cases of neonatal sepsis. (Table 2)

Of the 108/335 culture positive cases, band cells were observed in 95/ 108 cases (87.96\%). As the band cell ratio increased so did the culture positivity. Band cell ratio above $11 \%$ was found to correlate $100 \%$ with culture positivity. On analysis of the above data $\mathrm{P}$ value was found to be statistically highly significant. (Table 3)

Blood cultures when analysed showed that 227/335(68.05\%) blood cultures were sterile while 108/335 (32.24\%) were positive. (Table 4) Bacterial culture positivity was $67.76 \%$ and fungi $35.51 \%$. (Table 5)

Table 1: Distribution of Early and Late onset sepsis $(\mathrm{N}=335)$

\begin{tabular}{|l|l|l|l|}
\hline ONSET OF SEPSIS & $\begin{array}{l}\text { CULTURE } \\
\text { NEGATIVE }\end{array}$ & $\begin{array}{l}\text { CULTURE } \\
\text { POSITIVE }\end{array}$ & TOTAL \\
\hline Early onset sepsis & 123 & 36 & $\mathbf{1 5 9}$ \\
\hline Late onset sepsis & 104 & 72 & $\mathbf{1 7 6}$ \\
\hline
\end{tabular}

Chi square $=3.338, \quad \mathrm{P}=0.0004$ (highly significant)

Table 2: Distribution of Risk factors amongst subjects $(\mathrm{N}=\mathbf{3 3 5})$

\begin{tabular}{|l|l|l|}
\hline Risk factors & Present & Absent \\
\hline MSL & $130(38.8 \%)$ & $205(61.19 \%)$ \\
\hline PROM & $111(33.13 \%)$ & $224(63.87 \%)$ \\
\hline Mechanical ventilation & $27(8.05 \%)$ & $308(91.95 \%)$ \\
\hline Twin delivery & $10(2.98 \%)$ & $325(97.02 \%)$ \\
\hline
\end{tabular}

Chi square $=194.78$,

$\mathrm{P}=0.0001$ (highly significant) 
Correlation of blood culture and band cell ratio in neonatal septicaemia.

Table 3: Correlation of band cells with culture results

\begin{tabular}{|l|l|l|}
\hline BAND CELLS (\%) & $\begin{array}{l}\text { CULTURE } \\
\text { NEGATIVE }\end{array}$ & CULTURE POSITIVE \\
\hline $0-5$ & 47 & 24 \\
\hline $6-10$ & 4 & 25 \\
\hline $11-15$ & 0 & 28 \\
\hline $16-20$ & 0 & 15 \\
\hline $21-25$ & 0 & 2 \\
\hline$>25$ & 0 & 1 \\
\hline
\end{tabular}

Chi square $=60.00$,

$\mathrm{P}=0.0001$ (highly significant)

Table 4: Blood culture results

\begin{tabular}{|l|l|}
\hline NUMBER OF CASES & CULTURE REPORT \\
\hline $227(67.76)$ & Negative \\
\hline $108(32.24)$ & Positive \\
\hline
\end{tabular}

Figures in parentheses are percentages.

Table 5: Isolate wise distribution $(\mathrm{N}=108)$

\begin{tabular}{|l|l|l|}
\hline ISOLATES & NUMBERS & PERCENTAGE \\
\hline Bacterial & 69 & 63.89 \\
\hline Fungal & 39 & 36.11 \\
\hline
\end{tabular}

In our study fungal isolates contributed to about $36.11 \%$, of which Candida albicans was isolated in $23.08 \%$ and Non albicans Candida species were $76.92 \%$.

\section{Discussion:}

Neonatal sepsis is the most common cause of neonatal mortality despite treatment. Neonatal sepsis can be classified into two subtypes depending upon onset of symptoms. Signs of sepsis in neonates are often nonspecific and high degree of suspicion is needed for early diagnosis. Some laboratory parameters can be helpful for screening of neonates with neonatal sepsis, but none of it is specific and sensitive enough to be used singly. Delay of even a few hours in initiating treatment can considerably increase the morbidity and mortality.

Male preponderance observed in this study was similar to most of the other studies. (14-17) The high incidence of culture positivity in extramural cases could be due to unhygienic conditions, untrained (dai) nurse deliveries and premature deliveries being referred to our hospital from far off places. The culture positivity in intramural deliveries were owing to associated multiple risk factors and possible nosocomial infections.

Culture positivity of $22.64 \%$ in EOS and $40.90 \%$ in LOS falls into the similar range as observed by various other workers, reporting EOS ranging from 24-77.6\% and LOS ranging from 22.4-76\%. $(18,19,20)$ MSL, MVS and PROM as risk factors for development of sepsis were of great significance in the present study. It is thus important to keep a strict vigil in neonates falling into any of these categories.

Though blood culture is a gold standard for diagnosis of neonatal sepsis, but other factors like Absolute neutrophil count, Band cells, ESR, C-reactive protein, Procalcitonin and other serological markers are important in diagnosis of clinically suspected culture positive cases of neonatal sepsis. We found high percentage of band cells in culture positive cases of neonatal sepsis. This correlation was highly significant with band cell ratio above $11 \%$. We suggest that band cell count be used as a surrogate marker till the blood culture report is awaited. The limitation of this study was that blood culture for anaerobic pathogens was not performed so it is not fit to comment upon raised band cell ratio and culture negativity. It is thus recommended to put a parallel aerobic as well as anaerobic culture where possible.

The spectrum of organisms causing neonatal sepsis is quite different in developed and developing countries, like India. Both bacterial and fungal organisms are implicated in neonatal sepsis

The association between prematurity and blood borne candidial infections has been recognised for many years in the past. Over the same period of time the incidence of candidemia has escalated from 25 to 123 cases per 10,000 NICU admissions. Isolation of Candida species from a blood culture should never be regarded as a contaminant and should prompt an immediate search for evidence of dissemination, which occurs in approximately $10 \%$ of premature newborns with candidemia. (3) Despite the fact that fungal isolates which are often missed or considered contaminants also play an important role. A rising trend in pathogenicity of non albicans Candida is observed in our study.

Rates of bloodstream infection due to Candida species have increased steadily.(3, 4, 5)National nosocomial infection surveillance data of United States show that Candida species were the fifth leading cause of blood stream infection hospital wide and the fourth in the intensive care units. Current data from the SCOPE (Surveillance and control of pathogens of epidemiologic importance) surveillance system confirm that Candida 
species were the fourth leading cause of blood stream infection in United States $(21,22)$. An upsurge of previously ignored pathogens like non albicans Candida was also observed in our study.

\section{Conclusion:}

Neonatal septicaemia is one of the leading causes of mortality and morbidity in developing countries like India. In neonates, sepsis is difficult to diagnose clinically. They may be relatively asymptomatic until hemodynamic and respiratory collapse becomes evident. So, if there is even a remote suspicion of sepsis, neonates are frequently treated with antibiotics empirically until cultures are proven to be negative.

We recommend band cell ratio as a surrogate marker for neonatal sepsis to bridge the gap between suspicion and confirmation by culture results.

Continued surveillance of neonatal sepsis should be undertaken by hospitals at their levels due to changing scenario of the spectrum of organisms and changing pattern of antibiotic susceptibility. This in turn would act as a guideline in formulating rational empirical treatment strategy.

In resource-limited countries, hand washing or hand hygiene program still remains the most effective evidence-based strategy that will reduce the rates of nosocomial infection in NICUs. Fumigation of ICUs at regular intervals, avoiding overcrowding in ICUs and controlled patient nurse ratio would go a long way in reducing the morbidity and mortality due to neonatal septicaemia.

\section{References:}

[1] Nelson textbook of Paediatrics, $19^{\text {th }}$ ed. Philadelphia; Saunders publication, 2012. Thomson Press India Ltd.

[2] Tripathi S and Malik GK. Neonatal Sepsis: past, present and future; a review article. Internet Journal of Medical Update 2010 July; 5(2):45-54.

[3] de Haan TR, Beckers L, de Jonge RCJ, Spanjaard L, van Toledo L, et al. (2013) Neonatal Gram Negative and Candida Sepsis Survival and Neurodevelopmental Outcome at the Corrected Age of 24 Months. PLoS ONE 8(3): e59214. doi:10.1371/journal.pone.0059214

[4] Oberoi JK, Wattal C, Goel N, Raveendran R, Dutta S and Prasad K. Non- albicans Candida species in blood stream infections in a tertiary care hospital at New Delhi, India. IJMR 2012; 136: 997-1003.

[5] Singh RI, Xess I, Mathur P, Behera B, Gupta B and Misra MC. Epidemiology of candidaemia in critically ill trauma patients: experiences of a level I trauma centre in North India. JMM 2011;60: 342-348

[6] Ballot DE, Bosman N, Nana T, Ramdin T, Cooper PA. Background changing patterns of neonatal fungal sepsis in a developing country. J Trop Pediatr.2013 Dec; 59(6): 460-4.

[7] Ma CF, Li FQ, Shi L, Hu Y, Wang Y, Huang M and Kong Q. Surveillance study of species distribution, antifungal susceptibility and mortality of nosocomial candidemia in a tertiary care hospital in China. BMC infectious Diseases 2013; 13: 337

[8] Ng PC, Cheng SH, Chui KM, Fok TF et al. Diagnosis of late onset neonatal sepsis with cytokines, adhesion molecule and Creactive protein in preterm very low birth weight infants. Arch. Dis. Child Fetal Neonatal Ed 1997;77(3):F 221-7

[9] Avery's diseases of the newborn, 9th ed. Philadelphia; Saunders. Elsevier. 2012.p 544.

[10] Senzel LB, Spitzer ED. DeCristofaro JD. A Streamlined Laboratory Process for Obtaining Band Counts in Hospitalized Neonates. Lab Med. 2012;41(5): 279-283

[11] Bailey and Scott's Diagnostic Microbiology, $12^{\text {th }}$ ed. Philadelphia: Mosby Elsevier. 2007. Missouri press.

[12] Mackie and McCartney Practical Medical Microbiology, $14^{\text {th }}$ ed. New Delhi: 2007. Elsevier publication.

[13] Rex JH, Ferraro MJ, Anderson NL, Body BA, Forbes BA Fritsche TR et al. CLSI: Performance Standards for Antimicrobial Susceptibility Testing; Twenty-second Informational Supplement. CLSI document M100-S22

[14] Joshi SG, Ghole VS, Niphadkar KB. "Neonatal Gram negative bacteraemia.” IJP 2000; 67: $27: 32$

[15] Khatua SP, Das AK, Chatterjee BD et al. Neonatal septicaemia. IJP 1986;53 (4): 509-14.

[16] Jaswal RS, Kaushal RK, Goel A and Pathania K. Role of C-Reactive Protein in deciding duration of antibiotic therapy in Neonatal septicaemia. Ind. Paedtr.2003; 40:880-84.

[17] Tallur SS, Kasturi AV, Nadgir SD and Krishna BV. Clinico-bacteriological study of neonatal septicaemia in Hubli.IJP 2000;67(3): 169-74

[18] Kuruvilla KA, Pillai S, Jesudason M and Jana AK. Bacterial Profile of Sepsis in a Neonatal Unit in South India. Ind. Paed. 1998; 35: 851-858.

[19] Bhat RY, Lewis LES and Vandana KE. Bacterial isolates of early-onset neonatal sepsis and their antibiotic susceptibility pattern between 1998 and 2004: an audit from a centre in India. Italian Journal of Pediatrics 2011 37:32. http://www.ijponline.net/content/37/1/32.

[20] Sriram R. Correlation of blood culture results with the sepsis score and the sepsis screen in the diagnosis of neonatal septicaemia. Int J Biol Med Res 2011;2(1): 360-368.

[21] Jarvis WR, Edwards JR, Culver DH et al. Nosocomial infection rates in adult and pediatric intensive care units in the United States, 1980-1989. Am J Med 1991; 91(suppl 3B): 185S-91S.

[22] Edmond MB, Wallace SE, McClish DK, Pfaller MA, Jones RN, Wenzel RP. Nosocomial bloodstream infections in United States hospitals: a three year analysis. CID 1999; 29: 239-44. 